\title{
Viejas drogas, nuevos fármacos
}

\section{Old drugs, new medication}

\author{
Luis Jiménez Treviño*,**,***,****,*****, Angela Velasco****, \\ Julia Rodríguez-Revuelta*,**,***,****,*****, Pilar A SÁiz*,******,****,***** \\ *Area de Psiquiatría, Universidad de Oviedo, Oviedo, España. \\ **Centro de Investigación Biomédica en Red de Salud Mental (CIBERSAM), Oviedo, España. \\ ***Instituto de Investigación Sanitaria del Principado de Asturias (ISPA), Oviedo, España. \\ ****Servicio de Salud del Principado de Asturias (SESPA), Oviedo, España. \\ *****Instituto de Neurociencias del Principado de Asturias (INEUROPA), Oviedo, España.
}

$\mathrm{L}$ a historia nos ha demostrado, en los últimos años, cómo algunos compuestos diseñados originalmente para ser fármacos, han terminado formando parte del amplio abanico de drogas ilegales. Del mismo modo pero a la inversa, algunas drogas sintetizadas para un uso recreativo, han seguido el camino contrario y ahora forman parte del vademécum terapéutico.

Lo más habitual es el primer caso, productos de uso clínico a los cuales se les acaba dando un uso (y abuso) recreativo, ya sea por sus propiedades placenteras, estimulantes, psicodislépticas o entactógenas. Esto ha ocurrido con viejos conocidos en el mundo de las adicciones desde los tiempos de la morfina, originalmente analgésico, que dejó más de 400.000 adictos en la guerra de secesión, y se transformó en la droga de moda en la alta sociedad de finales del siglo XIX y principios del siglo XX. Posteriormente, los analgésicos opioides han seguido la estela de la morfina también en cuanto a su poder de abuso y adicción, especialmente a partir de una carta publicada en el New England Journal of Medicine en 1980, en la cual se minimizaba el potencial adictivo de los analgésicos opioides, y que pudo contribuir a la epidemia de adicción de analgésicos opioides en Norteamérica (Guardia Serecigni, 2018). Algo semejante ha ocurrido con la cocaína, también creada como producto anestésico e ingrediente habitual de elixires y tónicos de principios del siglo XX, que ha terminado constituyéndose en la segunda droga ilegal más consumida en Europa (Observatorio Europeo de las Drogas y las Toxicomanías, 2019), o las benzodiacepinas, consideradas una "epidemia silenciosa" debido al hecho de que un parte significativa de la población general española las consume bajo prescripción médica (Blasco-Fontecilla, 2018)

En el segundo caso, las drogas de abuso a las cuales se les acaba encontrando propiedades clínicas, cabría destacar al cannabis, la droga ilegal más consumida en el mundo, cuya incorporación al arsenal terapéutico de oncología en los últimos años no ha estado exenta de polémica (Hill, Palastro y George, 2019).

Pues bien, la actualidad nos obliga a escribir sobre una sustancia que estaría en un tercer caso, puesto que ha hecho el viaje de ida y vuelta: la ketamina

Desde su origen como anestésico, a mediados de los años 60 del siglo pasado, y tras incorporarse al mundo de las drogas de abuso a finales de los años 80 por sus efectos alucinógenos, la ketamina (así como su enantiómero esketamina) vuelve con fuerza al mundo de la terapéutica tras descubrirse sus propiedades antidepresivas y capacidad para reducir el riesgo de suicidio.

La ketamina es un agente anestésico no volátil sintetizado en 1962, y posteriormente comercializado para uso humano y veterinario. Se presenta como un líquido translúcido y es un derivado liposoluble de la fenciclidina (PCP). La ketamina se usa clínicamente como anestésico general, y está con-

Recibido: Octubre 2019; Aceptado: Noviembre 2019.

Enviar correspondencia a:

Luis Jiménez Treviño. Area de Psiquiatría. Facultad de Medicina. Julián Clavería 6 -3 º; 33006, Oviedo.

E-mail: luisjimeneztre@gmail.com 
siderado como un anestésico suave, de modo que su uso está más extendido en pediatría y geriatría. En nuestro campo, la psiquiatría, es uno de los anestésicos de uso habitual en las técnicas de terapia electroconvulsiva (Mion, 2017)

Sus características psicodislépticas fueron descubiertas después de que un gran número de pacientes informó de lo que sentían al salir de la anestesia. De hecho, los primeros en utilizarla como droga de abuso fueron los veteranos de la guerra de Vietnam, que habían experimentado sus efectos en los hospitales de campaña. Posteriormente comenzaron a aparecer casos de abuso entre los profesionales de la salud (al igual que ocurrió con la morfina y derivados). Los profesionales sanitarios que la utilizaron señalaban que una dosis muy menor que la anestésica, producía una experiencia psicodélica de gran intensidad. Sin embargo, su uso recreativo no se popularizó hasta mediados de los años noventa, coincidiendo con una caída de la pureza de la cocaína que se distribuía en los países occidentales, asociada a la cultura de la música electrónica y las "rave parties". La percepción de un bajo riesgo de su uso, la corta duración de su acción (comparada con otros alucinógenos), y su bajo precio, contribuyeron a que su uso se propagara rápidamente (Gómez-Arnau y Dolengevich, 2015).

En el mercado ilícito, la ketamina puede presentarse de muchas otras formas: líquido incoloro, polvo blanco (cristales blancos), comprimidos o cápsulas, por lo que es posible utilizarla por distintas vías de administración: intravenosa, intramuscular (líquido), rectal (líquido), nasal (polvo), pulmonar "fumada" (polvo) y oral (líquido, comprimido, o cápsulas). El preparado farmacéutico de ketamina que se presenta en forma líquida puede ser convertido en polvo mediante el simple método de "cocerla" o "cocinarla" a fuego lento. Este proceso se puede realizar utilizando un microondas, un horno a 90-95 $\mathrm{C}^{\mathrm{o}}$, o simplemente al «baño maría» hasta que se evapore el líquido. El resultante es un polvo blanco en forma de granos grandes de sal (cristales), que posteriormente se machaca para hacerlo más fino y apto para "esnifar". El polvo obtenido podrá venderse tal cual o puede, también, ser convertido en comprimidos.

Ha sido en los últimos diez años cuando comienza a plantearse el potencial de la ketamina como fármaco antidepresivo utilizando dosis subanestésicas. El primer estudio que sugiere esta posibilidad, data del año 2000 y es un pequeño ensayo, doble-ciego, con una pequeña muestra de 7 pacientes, basado en las teorías que sugieren un papel del glutamato en la etiopatogenia de la depresión (Berman, et al., 2000).

Durante los siguientes años se suceden los hallazgos que apuntan a un efecto antidepresivo de la ketamina así como un potencial efecto antisuicida y, curiosamente, muchos de estos datos, proceden de la observación de los efectos clínicos del fármaco en el contexto de su uso como anestésico en la TEC (Goforth y Holsinger, 2007; Okamoto, et al., 2010).
Estos datos preliminares han llevado a la comunidad científica a diseñar ensayos clínicos cuyos resultados han comenzado a ver la luz recientemente. Los datos que arrojan estos estudios muestran a la ketamina como un antidepresivo de acción rápida (horas), cuyos efectos clínicos incluyen la reducción de la ideación, y por tanto del riesgo suicida, al poco tiempo de recibir los pacientes la infusión de ketamina. Estos efectos, de todas formas, no son duraderos, y desaparecen a los pocos días de terminado el tratamiento experimental. La mayor parte de los ensayos utilizan ketamina en forma de infusiones endovenosas en entornos hospitalarios, lo cual supone un problema a la hora de generalizar el tratamiento, que quedaría relegado a un uso limitado a situaciones de gravedad en las que se requiera la hospitalización, ya sea total o parcial (Han, et al., 2016).

Afortunadamente, una vez se ha constatado este rápido efecto antidepresivo y el potencial antisuicida de la ketamina, la industria farmacéutica ha dado un paso más allá para facilitar un uso más amplio de esta alternativa terapéutica sintetizando una formulación intranasal, más accesible al uso clínico cotidiano que las infusiones endovenosas de ketamina, como es la esketamina.

La esketamina, enantiómero de la ketamina, y por tanto antagonista del receptor N-Metil-D-Aspartato (NMDA) que regula la transmisión glutamatérgica, se ha desarrollado como formulación intranasal para el tratamiento de la depresión resistente, consiguiendo una rápida reducción de los síntomas afectivos mayores, incluida la ideación suicida en pacientes con riesgo inminente de suicidio. El inicio de acción es tan rápido que se han observado efectos positivos del fármaco tan pronto como a las dos horas, y efectos clínicos relevantes en apenas 24 horas desde la administración intranasal de una única dosis (Slomski, 2019).

Los resultados de los ensayos clínicos en los que se utiliza esketamina como tratamiento añadido al habitual del paciente indican que, comparado con placebo, esketamina intranasal consigue una rápida mejoría de los síntomas depresivos, incluida la ideación suicida, en pacientes depresivos con riesgo inmediato de suicidio (Canuso, et al., 2018). La eficacia demostrada en los ensayos ha supuesto su aprobación por la Agencia del Medicamento de los Estados Unidos (FDA) como tratamiento coadyuvante para la depresión resistente en Marzo de 2019 (Cristea y Naudet, 2019) y por la Agencia Europea del Medicamento (EMA) en Diciembre de 2019.

El potencial del fármaco es tal, que la industria farmacéutica continúa avanzando en su desarrollo clínico, de modo que en el momento actual están en marcha más de 30 ensayos clínicos (10 de ellos en Fase III) de esketamina, tanto para el tratamiento de la depresión resistente, como para la depresión con ideación suicida, a corto y largo plazo (Fuente: clinicaltrials.gov). En la tabla 1 se recogen los resultados ya publicados de alguno de estos ensayos. 
Se abre, por tanto, una nueva vía en el tratamiento farmacológico de la depresión y las conductas suicidas. Quedaría por dilucidar, si este efecto antisuicida sólo se obtiene en el contexto del tratamiento y la mejoría de los trastornos afectivos, o por el contrario se trata de un efecto intrínseco del fármaco que permita su uso en la multitud de patologías mentales en las que existe un aumento del riesgo de suicidio. Es presumible que la investigación clínica nos aporte datos en este sentido en los próximos años.
Demos pues la bienvenida de nuevo a una sustancia, que comenzaba a ser demonizada por su potencial de uso y abuso recreativo, a la cual los avatares del avance en psicofarmacología, le han otorgado un papel prometedor en el tratamiento de los trastornos afectivos, y que puede contribuir a frenar al enemigo mortal de los profesionales que atendemos a pacientes con trastornos mentales: las conductas suicidas.

Tabla 1. Resultados publicados de los ensayos clínicos en depresión con Ketamina y Esketamina

\begin{tabular}{|c|c|c|c|c|}
\hline Fármaco & Referencia & Patología & Tiempo de estudio & Resultado \\
\hline $\begin{array}{l}\text { Esketamina, } \\
\text { spray nasal }\end{array}$ & NCT02133001 & Depresión mayor e ideación suicida & 4 semanas & $\begin{array}{l}\text { Mejoría en puntuación MADRS comparado con placebo } \\
\text { a las } 4 \text { y } 24 \mathrm{~h} \text {. Mejoría en ideación suicida a las } 4 \mathrm{~h} \text {. }\end{array}$ \\
\hline $\begin{array}{l}\text { Esketamina, } \\
\text { spray nasal }\end{array}$ & NCT01998958 & Depresión resistente a tratamiento & 10 semanas & $\begin{array}{l}\text { Mejoría en puntuación MADRS comparado con } \\
\text { placebo, mantenida en el tiempo de estudio }\end{array}$ \\
\hline $\begin{array}{l}\text { Esketamina, } \\
\text { spray nasal }\end{array}$ & NCT02493868 & Depresión resistente a tratamiento & 16 semanas & $\begin{array}{l}\text { Esketamina + Antidepresivo redujo el riesgo de recaída } \\
\text { en un } 51 \% \text { comparado con Placebo+antidepresivo }\end{array}$ \\
\hline $\begin{array}{l}\text { Esketamina, } \\
\text { spray nasal }\end{array}$ & NCT02417064 & Depresión resistente a tratamiento & 4 semanas & $\begin{array}{l}\text { Mejoría en puntuación MADRS comparado con } \\
\text { placebo. No se observó diferencias en eficacia entre } \\
\text { las dosis de } 56 \mathrm{mg} \text { y } 84 \mathrm{mg}\end{array}$ \\
\hline $\begin{array}{l}\text { Ketamina, } \\
\text { infusión I.V. }\end{array}$ & NCT02094898 & Depresión resistente a tratamiento & 4 semanas & $\begin{array}{l}\text { Remisión del cuadro clínico a la semana y } 4 \text { semanas } \\
\text { en el } 41 \% \text { de los pacientes }\end{array}$ \\
\hline $\begin{array}{l}\text { Ketamina, } \\
\text { infusión I.V. }\end{array}$ & NCT00088699 & Depresión mayor & 1 semanas & $\begin{array}{l}\text { Ketamina mejoró síntomas depresivos comparado } \\
\text { con placebo a los } 110 \text { minutos y a la semana de } \\
\text { tratamiento }\end{array}$ \\
\hline $\begin{array}{l}\text { Ketamina, spray } \\
\text { nasal }\end{array}$ & NCT01304147 & Depresión mayor & 24 horas & $\begin{array}{l}\text { Mejoría en puntuación MADRS comparado con placebo } \\
\text { a las } 24 \mathrm{~h} \text {. }\end{array}$ \\
\hline $\begin{array}{l}\text { Ketamina, } \\
\text { infusión I.V. }\end{array}$ & NCT01920555 & Depresión resistente a tratamiento & 3 días & $\begin{array}{l}\text { Mejoría en puntuación HAM-D y MADRS tras una } \\
\text { infusión de } 0.5 \text { o } 1.0 \mathrm{mg} / \mathrm{kg} \text { de Ketamina I.V. }\end{array}$ \\
\hline
\end{tabular}

Nota. clinicaltrials.gov

\section{Agradecimientos}

Este trabajo está financiado parcialmente por el Gobierno del Principado de Asturias PCTI 2018-2022 IDI/2018/235 con cofinanciación FEDER y por el Centro de Investigación Biomédica en Red de Salud Mental (CIBERSAM) y el Ministerio de Economía, Industria y Competitividad.

\section{Confilicto de intereses}

Pilar A. Sáiz ha sido consultora de y/o ha recibido honorarios/becas de Adamed, CIBERSAM, European Comission, GlaxoSmithKline, Gobierno del Principado de Asturias, Instituto de Salud Carlos III, Janssen-Cilag, Lundbeck, Otsuka, Pfizer, Plan Nacional Sobre Drogas and Servier.

\section{Referencias}

Berman R.M., Cappiello, A., Anand, A., Oren, D.A., Heninger, G.R., Charney, D.S. y Krystal, J.H. (2000). An- tidepressant effects of ketamine in depressed patients. Biological Psychiatry, 47, 351-354. doi:10.1016/s00063223(99) 00230-9.

Blasco-Fontecilla, H. (2018) Posmodernidad, sociedades adictivas, cannabis y comportamiento suicida: ¿Hacia un mundo feliz? Adicciones, 30, 3-8. doi:10.20882/adicciones. 1104 .

Canuso, C.M., Singh, J.B., Fedgchin, M., Alphs, L., Lane, R., Lim, P.,... Drevets, W.C. (2018). Efficacy and Safety of Intranasal Esketamine for the Rapid Reduction of Symptoms of Depression and Suicidality in Patients at Imminent Risk for Suicide: Results of a Double-Blind, Randomized, Placebo-Controlled Study. American Journal of Psychiatry, 175, 620-630. doi:10.1176/appi. ajp.2018.17060720.

Cristea, I.A. y Naudet, F. (2019). US Food and Drug Administration approval of esketamine and brexanolone. Lancet Psychiatry. Avance de publicación on-line. doi:10.1016/S2215-0366(19)30292-5. 
Goforth, H.W. y Holsinger, T. (2007). Rapid relief of severe major depressive disorder by use of preoperative ketamine and electroconvulsive therapy. The Journal of ECT, 23, 23-25. doi:10.1097/01.yct.0000263257.44539.23.

Gómez-Arnau, J. y Dolengevich, H. Ketamina. (2015). En H. Dolengevich (Ed.), Nuevas Drogas Psicoactivas. (pp. 229-241). Madrid: Entheos

Guardia Serecigni, J. (2018). Epidemia de sobredosis relacionada con la prescripción de analgésicos opioides en Estados Unidos. Adicciones, 30, 87-92. doi:10.20882/ adicciones.936.

Han, Y., Chen, J., Zou, D., Zheng, P., Li, Q., Wang, H.,... Xie, P. (2016). Efficacy of ketamine in the rapid treatment of major depressive disorder: a meta-analysis of randomized, double-blind, placebo-controlled studies. Neuropsychiatric Disease and Treatment, 12, 2859-2867. doi:10.2147/NDT.S117146.

Hill, K.P., Palastro, M.D. y George, T.P. (2019).Therapeutic cannabis use in 2018: where do we stand? Lancet Psychiatry, 6, 88-89. doi:10.1016/S2215-0366(18)30417-6.

Mion, G. (2017). History of anaesthesia: The ketamine story - past, present and future. European Journal of Anaesthesiology, 34, 571-575. doi:10.1097/EJA.0000000000000638.

Okamoto, N., Nakai, T., Sakamoto, K., Nagafusa, Y., Higuchi, T. y Nishikawa, T. (2010). Rapid antidepressant effect of ketamine anesthesia during electroconvulsive therapy of treatment-resistant depression: comparing ketamine and propofol anesthesia. The Journal of ECT, 26, 223-227. doi:10.1097/YCT.0b013e3181c3b0aa.

Slomski, A. (2019). Esketamine Nasal Spray Effective in Treatment-Resistant Depression. JAMA, 322, 296. doi:10.1001/jama.2019.10368. 